This report has been reproduced directly from the best available copy.

Available to DOE and DOE Contractors from the Office of Scientific and Technical Information, P.O. Box 62, Oak Ridge, TN 37831; prices available from (615) 576-8401.

Available to the public from the U.S. Department of Commerce, Technology Administration, National Technical Information Service, Springfield, VA 22161, (703) $487-4650$. 


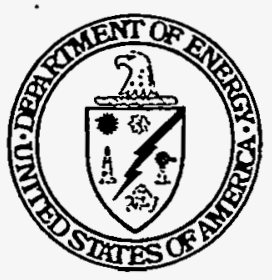

\section{Summary Results of an Assessment of Research Projects in the}

\section{National Photovoltaics Program}

\section{July 1995}

U.S. Department of Energy

Office of Energy Research Washington, DC 20585

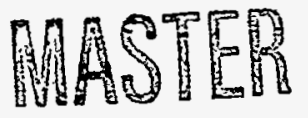

DISTRIBUTION OF THIS DOCUNEEN IS UNLIMITED

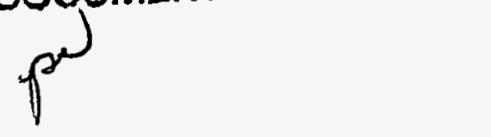





\section{INTRODUCTION}

The Office of Energy Research (OER) undertook an assessment of 115 research projects (1isted in Appendix A) sponsored by the National Photovoltaics Program. The Program is located within the Office of Energy Efficiency and Renewable Energy (EE). This report summarizes the results of that review.

The Office of Solar Energy Conversion is responsible for the management of the National Photovoltaics Program. This program focuses on assisting U.S. industry in development of fundamental technology to bring advanced photovoltaic energy systems to commercial use.

The purpose of the assessment was to determine the following:

1. the quality of research of individual projects,

2. the impact of these individual projects on the mission of the program, and

3. the priority of future research opportunities.

The assessment was conducted using the quantitative methodology developed by OER for assessing the quality of research and development programs within the Department of Energy (DOE). This methodology is described in Procedures for Peer Review Assessments, D0E/ST-0007P. At the time of this review, it had been used by OER in 30 prior assessments of over 2,600 projects in various DOE research programs.
The 115 research projects were reviewed by 15 panels of experts in review sessions held in Denver, Colorado, in August, October, and November of 1994, and in Bethesda, Maryland December 1994. These projects are Tisted in Appendix A. The reviewers in each panel were expert in the technical area (e.g., photovoltaic module development and applications) addressed by the 7-10 projects assigned to that pane7. The reviewers participating in this assessment are listed in Appendix B.

Rating factors included project scientific and technical merit, mission relevance, appropriateness and level of innovation of the technical approach, quality of the project team, productivity, and probable impact on the program's mission. Each project was also given an overal1 evaluation supported with written comments.

\section{Quality of the Projects Reviewed}

A project's overall evaluation was rated on a scale from 0.0 to 10.0 , with numerical values corresponding to a qualitative descriptor (e.g., outstanding, strong, good, needing management attention, or having serious shortcomings). Project overall evaluation ratings of 5.0 to 10.0 signify project quality ranging from good to outstanding. Descriptors below $\mathbf{5 . 0}$ identify projects with deficiencies or serious shortcomings that particularly warrant further review and attention from program management. 
Figure 1 on the following page summarizes the project overall evaluation ratings established for the 115 projects reviewed by the 15 panels. Figure 2 Summarizes the results of a similar review conducted in 1989.

A total of 102 projects (89 percent of those rated) were judged to be good or better (i.e., had project overall evaluations of 5.0 or higher). of these projects, 57 projects (50 percent of those rated) were judged to be strong (i.e., rated 7.0 to 8.9 ) and 5 projects were rated excellent. Thirteen projects (11 percent) reviewed project overall evaluation ratings lower than 5.0.
The reviewers judged these to be projects with some deficiencies, or serious shortcomings, that need to be addressed by program management.

Compared to the photovoltaic peer review assessment conducted in 1989, the percentage of projects rated strong (i.e., 7.0 to 8.9) increased and the percentage of projects in need of management attention (i.e., 3.0 to 4.9) decreased. Thus, the National Photovoltaic Program's project ratings have improved since the review 5 years ago. A higher percentage of the Program's projects are rated good to outstanding (5.0 to 10.0) than in the typical program reviewed by Office of Energy Research. 
Figure 1

\section{Assessment (115 Projects)}

50

$50 \%$

40

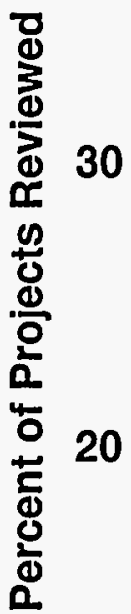

10

$10 \%$

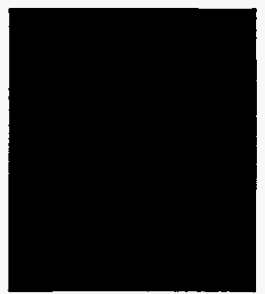

3 to 4.9
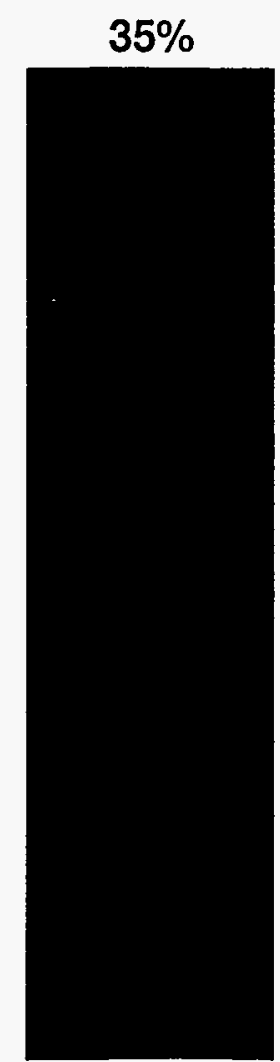

5 to 6.9
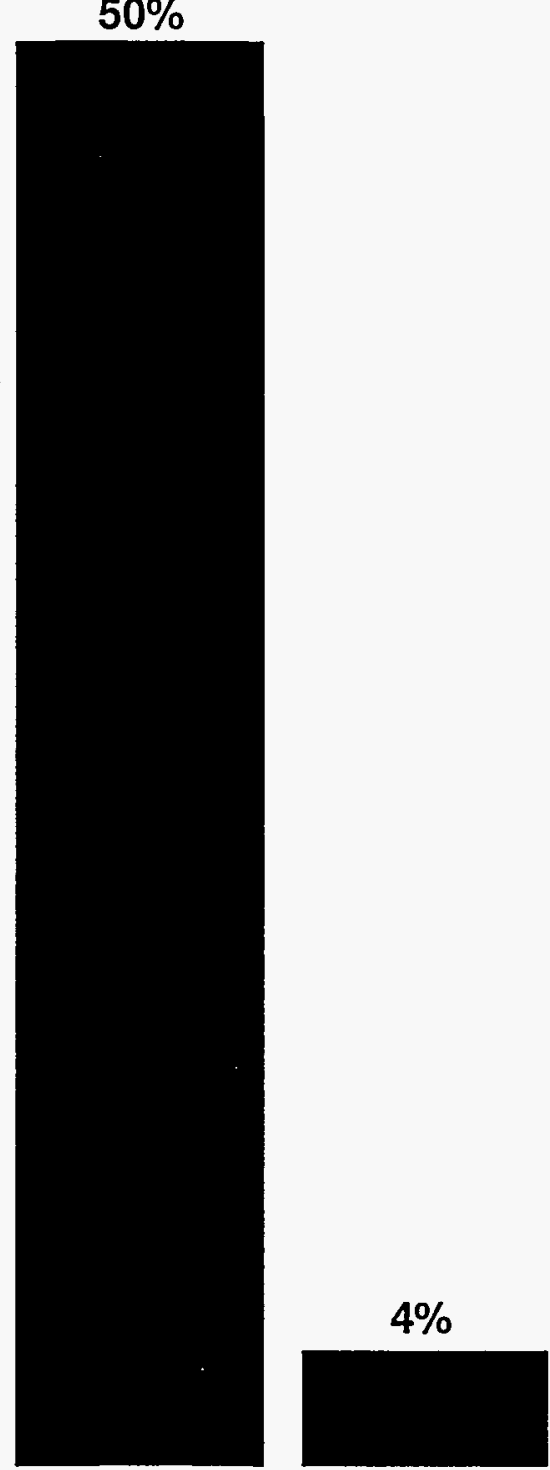

7 to 8.9

9 to 10

Overall Ratings

Note: An outstanding project would rate 9-10. A strong project deserving of continuing priority support would score $7-8.9$, while a good project with negligible important shortcomings would receive a score of 5-6.9. A good project with some deficiencies or concerns that should be addressed would warrant a rating of 3-4.9, and a project with serious shortcomings which warrants close reevaluation would rank 0-2.9. 
Figure 2

\section{Assessment (129.Projects)}

50

40

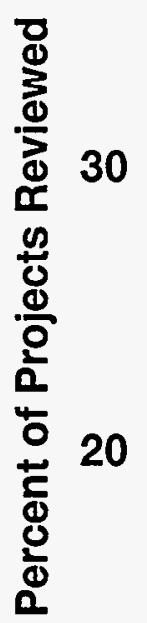

10

$14 \%$

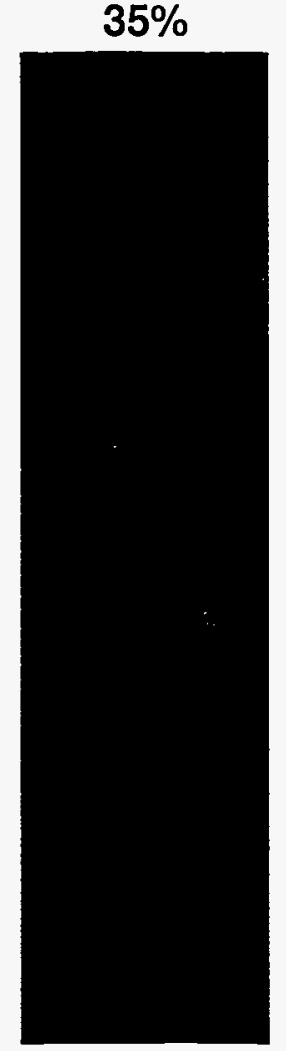

5 to 6.9

$46 \%$

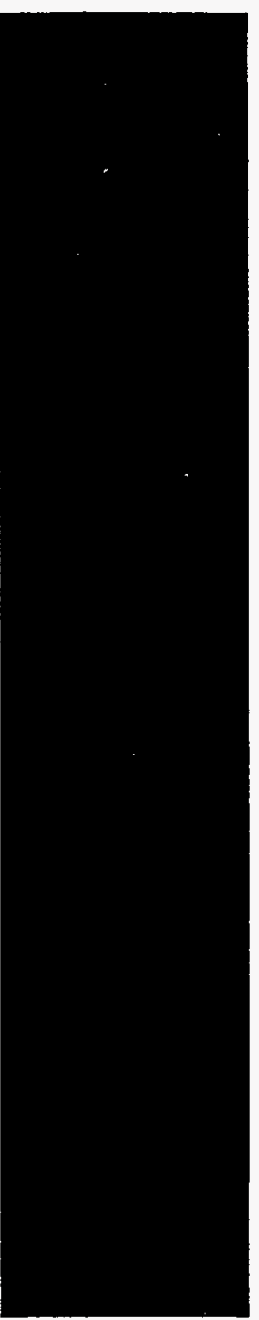

$4 \%$

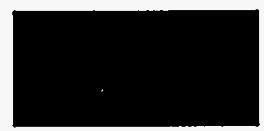

0 to 2.9

3 to 4.9

Overall Ratings

9 to 10

Note: An outstanding project would rate 9-10. A strong project deserving of continuing priority support would score 7-8.9, while a good project with negligible important shortcomings would receive a score of 5-6.9. A good project with some deficiencies or concerns that should be addressed would warrant a rating of 3-4.9, and a project with serious shortcomings which warrants close reevaluation would rank 0-2.9. 


\section{FUTURE RESEARCH OPPORTUNITIES}

Described below are the research opportunities identified by the reviewers in the general area of photovoltaics (PV) for power applications.

Review Topic 1 - Photovoltaic Manufacturing Development (PVHaT)

\section{High Energy Concentrator System Development}

The ENTECH concentrator did not address the well-documented high efficiency attainable from specially designed cells operated at high concentrations. The effects of a 50 percent efficiency increase above the 13-14 percent level attained by ENTECH on reduced balance-of-plant, hence levelized energy costs, are very significant.

A multi-year effort to develop the next generation concentrator could substantially improve the probability of achieving the DOE goals. An integrated design and manufacturing effort would be required to effectively couple the demands of the high efficiency concentrator cell with the module and tracking system.

Development of a Flexible High Throughput Solar Cell (Substrate) Cutting Machine

Most solar cell manufacturers process wafers or substrates that require a sizing operation. Various high-speed cutting processes have been developed by other industries to perform similar functions.

An evaluation of these cutting processes and selection of a generic automated approach that could integrate one or more of these processes in a flexible manufacturing operation would benefit the solar industry.

\section{Manufacturing Development for Complete PV Systems}

PVMaT work to date has focused on module manufacturing and generic manufacturing issues. The PV industry and PV users could benefit from project activity aimed at design/manufacturing/assembly of complete PV systems that perform a specific market-driven function or service. Projects could be funded that support cost-shared efforts among manufacturers and users to develop, manufacture, and demonstrate such complete systems.

\section{Transparent Encapsulants}

All photovoltaic technologies share a common requirement for transparent encapsulant materials. These materials, usually organic (elastomers and plastics) or inorganic (glasses) polymers, are usually selected from existing products based on the degree to which their physical and chemical properties match the requirements of the PV industry. Factors such as cost, toughness, stability, transparency, etc., are balanced to optimize system cost effectiveness even though such transparent encapsulants constitute a significant cost component of deployed photovoltaic systems. They have rarely been developed on the basis of their applicability to this technology. This is so because they are manufactured on such a large scale that the PV industry's requirements provide little leverage for inducing the required product developments or improvements. 
The perceived opportunity is to develop improved transparent encapsulants with properties which meet the requirements of the PV industry. better than do existing, commercially available materials. In particular, the objective would be to provide sufficient resources to attract a team with research capabilities appropriate to the development of new organic and inorganic polymers for PV applications. Such a team might exist in a university laboratory with capability of glass formulations and surface treatments or in the research laboratories of the large glass and plastics manufacturing companies.

\section{PV Manufacturing Toolbox}

There exists today many toots, methods, techniques, and fields of expertise that are commonly used in the IC manufacturing industry to optimize manufacturing costs, throughput, and cycle time. These same tools are used to increase the manufacturing process robustness which has a direct effect on profitability. These same techniques should begin to be applied to the PV industry through appropriate technology and knowledge transfer programs. These tools and methods include the following:

1) yield enhancement;

2) reliability analysis applied to:

thin film cells adhesion/delamination in modules or thin films, stress induced degradation material interactions, corrosion/inter-diffusion stability fundamentals, acceleration aging, and testing;
3) SPC applied to cell, module, and system manufacturing;

in-process inspection

Taguchi analysis

4) fundamental research on mechanisms of degradation; and

5) CIM implementation.

The implementation of the above techniques will help develop high quality and long term reliable products, encourage some level of standardization, and low cost manufacturing.

\section{Low Cost Silicon Feedstock for Crystalline Silicon Solar Cells}

A major cost ingredient of present-day crystalline silicon (1-sun) solar cells is the feedstock used to create the silicon blanks. For this application, the PV industry typically employs "offspec" material which is a by-product of semiconductor silicon manufacturing. But the availability of this material is limited and, in time, witl not be available in sufficient quantity to satisfy the needs of a large-scale solar industry. The manufacturers of silicon feedstock for the IC industry have little incentive to change their technology to meet the needs of the solar industry.

Two facets of research and development could be sponsored to create a "solargrade" silicon for solar applications.

1) Under DOE sponsorship in the 1970 's, several alternative approaches to low-cost "solargrade" silicon were investigated, but no standards were developed to quantitatively define "solar-grade." Techniques for analyzing 
extremely low levels of impurities such as tungsten, molybdenum, chromium, and other life-time killing impurities (including neutron activation analysis, deep level transient spectroscopy, and solids mass spectrometry) need to be developed as monitoring tools, to provide a quantitative definition of "solar-grade" silicon.

2) A re-evaluation of the alternative approaches for producing low-cost silicon feedstock needs to be undertaken, using these solargrade standards as a criterion. Processes need to be reexplored, costed, and evaluated for application in the solar industry.

A project such as this could make available a low cost silicon feedstock that does not depend on a by-product material of Timited availability.

Review Topic 2 - Photovoltaic Applications and Market Development

\section{International}

The DOE Photovoltaics Program has a number of opportunities for enhancing the development of international market access and growth.

The World Bank, Global Environmental Facility, and Asian Development Bank have expressed interest in activities that will assist the expansion of renewable projects. The Department of Energy could step-up its role in assuring that this interest results in availability of capital.

\section{Visiting Professional Program}

The National Renewable Energy Laboratory (NREL) and Sandia presently have an open door to worldwide scientists and engineers permitting side-by-side working experiences with lab specialists. A corollary program could be created by simply inviting professionals from developing countries to work side-by-side with national laboratory employees now addressing market development tasks. In so doing, personal relationships are established between the professionals who may staff developing area energy planning teams and the experts in the labs. As a consequence, the $1 \mathrm{ab}$ insight to the needs in developing countries is expanded, and the visiting scientist or engineering professional has a U.S.-based contact who can supply references to sources of U.S.-produced goods and services.

\section{Rural Electrification Models}

As a result of the diversity of field experience shared between NREL and Sandia with a variety of repayment techniques, it is time to consider summarizing the knowledge from these pilot efforts and quantifing the financial opportunities. For example, in Brazil, the state transmission and distribution utilities are responsible for field deployment, maintenance, and monthly fee collection. In Honduras, local cooperatives have this responsibility. Other models include involvement of National Rural Electric Cooperatives, agricultural cooperatives, leasing programs, credit programs, and tolling fees. These various approaches may be privately financed or supported by various international lending agencies. It is time to evaluate these early field experiences for their strengths and weaknesses. From these insights it may be possible to gain new tools for 
project optimization in different regions.

The NREL and Sandia would then provide a major service to the international arena by simply assembling a "score-card" to track the number of solar home systems installed by country. While challenging to assemble and maintain, this would result in a quick guide to determining where solar programs are succeeding and to what degree. This information would be useful to a wide variety of people from banks to energy planners to end users.

\section{International Competition and Collaboration}

Lending institutions need to be convinced that wealth can be generated through investment instruments that are designed for renewables. The DOE may consider taking the lead in pulling together a unified approach by U.S., European, and Japanese agencies in support of this goal.

In juxtaposition to this is the need for the U.S. to have a proactive and on-going competitive benchmarking effort to identify and define the strategies being developed in Europe and Japan. The effort would assist U.S. manufacturers of PV goods and services to compete in international markets.

\section{Hybrid Power Systems}

One major opportunity of note to emerge from the review of the Brazil and Department of Defense projects is the vast market potential available today for PV-diesel hybrids. Many diesel power units are being subsidized, suffering from lack of spare parts and local repair resources and difficult fuel delivery. DOE could help provide the tools to access this market, as a priority.

\section{Activities to Increase Market Penetration of PV}

The rural electric cooperatives (RECs) represent a large market for PV systems. The technical staff of the typical REC is small, and the national labs can help with their education and training in the appropriate use of PV systems. Thousands of PV systems could be installed in cost effective applications, and the experience gained could serve as an important link to the overall utility market.

Continuation of the effort to improve the reliability of PV systems and balance of system (BOS) components merit inclusion in the utility market expansion program. System integration issues should continue to be addressed until overall reliability reaches a level acceptable to the electric utilities.

\section{Government Agencies}

Government agencies (local, state, Federa1) represent a significant market for photovoltaic technology. Many cost effective applications exist today. Other applications are longer term and may require further research and development and/or financial subsidies for pilot projects. Major needs in furty developing this market are: 1) education, 2) identification of applications, and 3) technical and financial assistance. Additional effort in the following areas would benefit the opening of the Government agency market:

1. Life Cycle Cost Analysis: Current agency regulations for performing life cycle cost (LCC) analysis sometimes exclude the use of "external" factors like environmental and societal costs. These costs can be very real. For 
instance, with any diesel generator power system there is the possibility of a fuel spill with the attendant environmental damage and clean-up costs. Fuel spill clean-up costs are high and represent a significant potential savings for photovoltaic projects. A DOE field of effort could be to perform studies to identify and quantify these "externalities" and help facilitate application and uniformity of LCC analysis methods throughout Government agencies.

2. Design Assistance Center Type Efforts: Current Design Assistance Center (DAC) type efforts include education and outreach, technical assistance, and pilot project support. These efforts are critical to further market identification and development. Consideration should be given to expanding the on-going efforts. Careful attention should be paid to close coordination of DOE National Lab efforts in these areas to make most efficient use of resources, (people facilities, and funding).

3. Industry Involvement: The National Labs can be key facilitators in the industry/end user relationship. Processes and methods to improve the communication between the industry and the user merit identification and facilitation by the National Labs.

4. Procurement Barriers: Institutional barriers involving procurement of photovoltaic systems are formidable. Effort could be sponsored by DOE to facilitate streamining and uniformity of procurement processes.
Review Topic 3 - PV/Buildings and Demand-Side Management

\section{Standardized PV Component Technology}

Research and development are needed to evolve standardized PV components which can be used to make installation of a wide variety of PV-DSM (Demand Side Management) and PV-building systems easier and more commonplace. This family. of components would include PV modules, power conditioning units, other electrical equipment, and mounting structures. The components need to be compatible in terms of both physical and electrical characteristics to allow easy build-up of PV systems of varying size and configuration with minimal design and little or no engineering effort. They should also be designed for suitable safety and conformity to existing and anticipated code requirements. Finally, the design of standardized components to ease future 0\&M burdens is very important.

The availability of standardized PV component packages would result in increased PV market penetration due to: 1) simplification of the effort, time, and cost associated with deploying PV systems in building applications; 2) allowing a higher degree of mass production of components which also serves to lower cost; and 3) facilitating additions and removal of components. The increased market penetration resulting from this project's results would occur in both utility and non-utility markets.

\section{Low Cost Storage}

A 1arge number of PV applications rely on batteries for storing the solar energy. Currently, the selection of batteries for PV applications is limited, and their characteristics (electrical, physical, and logistical) 
are often less than desirable, adding cost, inconvenience and problems to the projects. This research suggestion contains four ideas whose implementation could help remove some of these impediments and substantially lower the cost of storage in a PV system. These ideas are:

\section{1) Battery Integrated in Building Environment}

There is a need to understand more fully how batteries are currently used in buildings. An effort should be undertaken to evaluate and disseminate information on how batteries can be located, what constraints they pose in buildings, and whether there is any compatibility between PV's and batteries already used for building applications, such as in the Uninterruptable Power Supplies and emergency lighting systems. Batteries for PV installations could be designed or selected for the building environment and structure to produce a longer $7 \mathrm{ife}$ and lower cost. For example, for batteries placed inside a building, it is possible to maintain the temperature of the battery system within the optimum range for long-life and efficient operation.

\section{2) Reliable Batteries for PV Systems}

The idea is not specific but suggests that an effort be made to develop a battery with performance characteristics specifically for PV applications.

\section{3). Low Cost Storage}

Where batteries are not cost effective for specific PV systems, a]ternative storage methods should be explored, for example, thermal storage, especially in building applications.

4) Analytical Tools for Batteries

Since batteries used in PV systems experience variability in charge and discharge amount and duration, additional knowledge of the dynamic and static characteristics is needed to quantify battery performance and 1 ife prediction.

It is suggested that suitable types of batteries be dynamically exercised and their current and voltage characteristics be examined and classified under PV applications. Batteries could then be designed around optimization based on the measured dynamic characteristics, or, at least, selected to satisfy the requirements suggested by those characteristics. Such information could also be supplied by battery manufacturers so that such batteries could be tested in the field.

Application Specific Components and Packages

A focused effort to develop replicable, high volume, applicationspecific components and complete system packages would be a, valuable use of resources at this time.

Commercialization efforts such as TEAMUP, PVBONUS, State PVAU initiatives and SMUD'S PV Pioneer Program could then procure these components and systems at a lower cost as volume grows. Some example components and packages would be: 1) residential roof installations, 2) commercial building facades, 3) parking structure vehicle charging systems, 4) alternating current modules, 5) packages for various building types, and 6) mobile structures. 
The existence of such a common set of products which are application specific encourages collective buying, thereby contributing to lower cost.

Review Topic 4 - PV Systems Evaluation, Standards and Codes

\section{Standards Development}

PV technology and component development is changing rapidly. Standards activities which attempt to cover this technology are in need of continuous update. Some basic standards covering PV components, systems, and applications have been published in recent years with DOE assistance. Because PV systems sales are growing rapidiy, the need for timely standards is growing. The scope of needed standards is also expanding, especially in the areas of PV systems, safety, reliability testing and in related fields such as battery storage.

A large fraction of PV products manufactured in the U.S. are exported. For that reason, compliance with and development of international standards is taking on greater importance. There is a constant and expanding need for U.S. experts to participate in international PV standards development. Because participation in standards development in the U.S. is voluntary, DOE support of these activities is essential.

The development of domestic and international standards have been partially supported by DOE with industry providing a share of the cost. Because the PV industry is not yet financially stable, DOE's leadership is essential for continuity of standards efforts.
The DOE has provided both financial support and assistance with technical and administrative experts who participate and have Teadership positions on standards committees. This support and leadership role by DOE could be expanded to assure adequate coverage of the growing needs in both the domestic and international standards development.

\section{Economic Analysis}

A uniform methodology for Multilateral Development Banks and utilities to use in comparing grid extension with local diesel generation and renewable power systems is needed.

Due to differences in tax treatment, tariff structures and competition between short-term cash needs and long-term quality investment, balanced planning for power systems is an increasing need. In many cases, PV and other renewables can already meet or beat the price performance of competing technologies. However, conventional lending institutions do not have a convenient tool to make decisions about investment-grade opportunities in the renewable field.

The approach would be to assemble a team with representatives from the utility industry, renewable system suppliers and lending institutions. Representation would include traditional capital sources such as GE Capital, Bear Stearns, or equivalent organizations. This team would be tasked with producing a tool for use by energy planners in selecting the optimum power system. A model for this approach could be the one currentiy used by utilities for placing a value, derived from a list of benefits, on PV systems used at substations and distributed applications. 
A financially-based software tool with uniform standards for technology intercomparison with sensitivities to discount rate and portfolio planning, such as advocated by Shimon Auberuch, would be integrated into one easy-touse package. The design options would range from applications as large as substation support to those as small as individual solar home lighting systems on a country-wide basis.

\section{Market Development}

The Department of Energy can assist in new market development by providing seed funding for sustainable projects such as has been accomplished in Brazil with World

Bank encouragement. The result is that large quantities of U.S.produced PV products are reaching numerous international users. The long-term systems evaluation experience, which is being continuously assembled as an integral part of the on-going DOE PV program, is useful to assuring the quality and longevity of such important worldwide initiatives. A creditable track record in this area will enhance growth of the PV industry and result in steady cost reduction.

Internet Based Knowledge Acquisition Delivery System for Photovoltaics

A centralized clearinghouse for the acquisition and delivery of knowledge in the field of photovoltaics is proposed. This will be based on the internet. It will provide opportunities for posting information by the provider and requestor of services. These services could include information about applicability of PV for specific needs and the types of appropriate PV systems and components that could be used to achieve the goal. Relevant information about possible financial packages to achieve these goals would also be included.

In addition to bringing together requestors and providers of services, this medium can be used to disseminate information about PV related training, workshops, short courses being offered in the country and internationally. Information about satellite hookupbased education and training opportunities can be provided through these means.

The electronic medium to be used for this purpose will be internet based but will take advantage of various options available in internet. This will include gopher, world-wide web, mosaic, and simple bulletin boards. This will allow users with different levels of hardware and communication capabilities to access the relevant information.

Electronic database and information access means provided by various bilateral and multilateral donor agencies can be linked to this Knowledge Acquisition and Delivery Network for PV (KADNPV) that could be developed quickly and in a cost effective way. Linkages with many existing systems will facilitate quick accesses to this system by a large number of users in the United States and overseas.

\section{Systems Testing and Evaluation}

A number of PV systems of all sizes have been installed over the past 5 to 10 years. However, the long term performance or test results are either not available or not consistent to allow useful analyses or meaningful conclusions. It is, therefore, extremely important to try to standardize the type of data required in order to develop appropriate comparative criteria and recommendations for future 


\section{improvements.}

The following activities can be pursued to accomplish the above objectives:

1) Continue testing and evaluation of existing PV systems to determine: a) performance, b) maintenance requirements, c) reliability of system and components (including BOS), and d) identification of troublesome components.

2) Collection and analysis of operational data of systems located throughout the world. Identification of unique problems and component successes and failures. (Routine calibration is necessary to maintain accurate data.)

3) Insta7lation and testing of new systems incorporating the most promising (advanced) components with promise for cost reduction. Testing and evaluation of such systems to establish system and component performance under real 1 ife operational conditions.

\section{Component Evaluation}

The performance and reliability of PV systems is affected by all the system components (PV modules, structure, wiring, controls/ power conditioning, energy storage, and specialty load devices). For the cost and performance of PV systems to improve, all of the components need to jointly evolve to assure compatibility. Often, improvements in the characteristics of one component will affect the other components.

Many of the power conditioning and energy storage needs of the PV program are shared or complemented by other technologies such as electric vehicles, advanced lighting, and UPS power systems.

Evaluation of components for PV power systems would be more effective if undertaken in an environment that includes inputs from these other complementary technologies. Effective use of the evaluation requires that the results of the evaluation be incorporated as product improvements and that the technology thus developed be widely available.

Evaluation of components can be either laboratory and/or field application based. Efforts must be made to evaluate products from all international sources and disseminate the results.

\section{Identification and Removal of Hardware Barriers to PV Systems Commercialization through CRADAs}

Utilize the data collected from systems and component evaluation to identify new products or improvements to existing products that would improve system performance and lower cost. It is important to close the loop so that lessons learned result in system improvements. Provide this information to industry and work with industry to quickly commercialize these new products.

Key components of this activity include: 1) distillation of system and component evaluation data to identify opportunities for new and improved products, 2) dissemination of this "knowledge" to industry through CRADAs or equivalent mechanisms, 3) solicit input from industry ideas for new products, and 4) assist industry to bring these new products to market by providing technical and or financial assistance. 
The types of products that may result from this activity include: inverters (stand-a-lone, hybrid, and grid tie); system controls; system wiring, circuit protection, and interconnection components; and support structures and trackers.

Review Topic 5 - Photovoltaic Module Development and Evaluation

\section{Module Reliability and Durability}

There are two major areas in which research is needed. The first major area is module reliability and durability. This is divided into three sub areas:

1) correlation of accelerated lifetime test results with actual expected module life,

2) determination of environmenta] effects on modules, and

3) broadened work on encapsulant.

The work on module durability and reliability would be the basis for better understanding the broad spectrum of degradation mechanisms that lead to module failure. Improved module technology would be developed which leads to lower cost via longer life in support of longterm DOE goals.

\section{Concentrator Development}

The second major area of needed research is increased concentrator technology development and industry support. This would include tests pertaining to current concentrator components, modules, and systems.

The concentrator research would assure that an important group of PV technologies (low, medium, and high concentration) are better understood with respect to different approaches. Any of several PV concentrator concepts could be a rapid path to substantial reduction of PV costs.

Other areas considered were:

1) ongoing assessments of PV technotogies, and

2) evaluation of benefits derived from site radiometric data.

Review Topic 6 - PV Measurements and Characterization Technique Development

Interface Characterization with offAxis Electron Holography

Electron holography performed in a transmission electron microscope with a coherent source of electrons (a field emission gun) is a new method that can enable quantitative measurement of the contact potential at interfaces in photovoltaic devices with nanometer resolution, a previousiy unobtainable goal.

The method has been developed, and the variant that is most promising at this moment is off-axis electron holography. In this method, the coherent incident illumination is split into a reference wave which passes through vacuum and an object wave which passes through the specimen (i:e., the interface). The electrostatic potential at the interface changes the energy of the electron, thus changing the wavelength within the specimen, and that corresponds to a phase shift which is proportional to the potential. When this object beam is superimposed with the reference beam, the information about the potential is encoded in the interference pattern. This pattern is a hologram, and when it is reconstructed, the phase image maps the spatial distribution of the 
potential at the interface. The method was demonstrated for a field outside of $S i p-n$ junction in 1985 (Pozzi, et a1., PRL) and this year at the $S i p-n$ junction (ASU group, Maccartney, et al., First International conference on electron holography, Knoxville, September 1994).

The DOE has one such laboratory at Oak Ridge (High Temperature Materials Laboratory). A more directly related development is happening at AT\&T Bell Labs in the group led by Ourmazd.

\section{Near Field Optical Scanning Microscopy}

The trend toward polycrystalline thin film materials requires the use of techniques capable of investigating the optical properties of the material on a grain-by-grain basis. Cathodoluminescence studies at NREL (Matus) show striking differences in the optical properties of different grains. An alternative highresolution technique for the study of optical properties is near field optical scanning microscopy. This new technique overcomes the diffraction limit by utilizing the evanescent fields from an illuminated optical fiber that is brought to a close distance from the sample surface. The approach is similar to scanning tunneling microscopy, but the use of light, particularly for PV materials, opens up exciting possibilities for spectroscopy with a Tateral spatial resolution of a few tens of nanometers.

\section{Scanning Force Microscopy}

Non-contact mode scanning force microscopy can map forces and force gradients relating to various physical interactions (Van der Waals attraction, electrostatic/magnetic forces) between tip and sample in close proximity with lateral resolutions of the order of tens of nanometers. As nanoscale characterization of polycrystalline PV materials proceeds, it will become useful to correlate structural and compositional effects with the Tocal surface photovoltage. This can be probed by illuminating the sample while mapping electric fields and near-surface equipotentials directly. Such measurements can be compared to standard macroscopic measurements of surface photovoltage that average over large dimensions.

\section{U1tra Violet (UV) Reflectance Spectroscopic Ellipsometry}

UV reflectance spectroscopy is very sensitive to grain size, orientation, and other physical parameters of thin film PV materials that determine their quality. The technique consists of measuring the optical reflectance from the film surface within a wavelength range where the band structure of the material contains one or more critical points. In this energy range the band structure is highly sensitive to any perturbations in the lattice. Dislocations, grain boundaries, stress, etc., have very large effects on the band structure and therefore on the reflectance spectra.

The optimum method for making this measurement is to use a spectroscopic ellipsometer. The addition of polarized light to the measurement allows the real and imaginary parts of the dielectric function to be separated, giving the refractive index and absorption coefficient spectra over the measured range. However, measurements using a simple spectrometer with good resolution are quite adequate. Normally the wavelength of interest is in the ultraviolet range between 3 and $5 \mathrm{eV}$,
0.2 microns
to
0.4
microns 
wavelengths. The optimum range will

- depend on the material being studied.

Low Cost, Rapid, Measurement and InLine Process Control Equipment for PV Manufacturing

The PV industry comprises a number of small companies that are unable to make large capital equipment investments for performing diagnostic measurements for research and development and in-line statistical process control (SPC). SPC is a mandatory tool within the PV industry where yield, performance, and cycle time are of importance, and manufacturers would benefit from a suite of low-cost analytical and characterization tools developed specifically for PV industrial application.

The requirement is for: identifying the need for specific measurement and characterization tools within industry, 2) developing - low-cost tools to meet these needs, and 3) providing industry with the information to fabricate the equipment or to purchase it from equipment manufacturers. An increased thrust in this area would be of major benefit to the PV industry as a whole.

Review Topic 7 - Crystalline Silicon PV Materials and Defect Characterization

\section{Process Modeling}

There is a need for user-friendly software for process modeling. This software should be based on existing process models, but even so, a major effort will be required to do this. This is essential in order to make process models useful and widely available. It is also important that a standard computer platform be established for process modeling to facilitate interchange and compatibility of software.

\section{Substrates for Thin-Film Deposition}

Creating thin multicrystalline Si films on substrates by techniques such as LPE appears promising as illustrated by the non-optimized test cells with 15 percent efficiency. If future results should show continued improvement to the point where commercialization of the technique looks promising, research to optimize substrates (performance $v$. cost) is needed. This research should investigate factors such as: 1) outdiffusion of impurities from the substrate into the film, 2) identification of outdiffusing species and rate of outdiffusion, 3) design of barrier layers to minimize out diffusion, 4) integrity requirements on barrier layers such as pin holes and pin hole density, and 5) stresses caused by different thermal expansion coefficient of $\mathrm{Si}$ and the substrate and the influence of these stresses on the performance and long-term stability of solar cells fabricated.

\section{CVD Processes for Multicrystalline} silicon

The use of CVD to fabricate multicrystalline $S i$ films is not currently being investigated. CVD is overwhelmingly the technique used to deposit polycrystalline films. A very large technological and equipment manufacturing base is available. A large program to generate polycrystalline thin-films on glass substrates with large (but not too large) grain size has been developed in Japan to provide the starting material for the next generation of active matrix circuits in flat panel displays. 
A project to generate thick multicrystaliine $\mathrm{Si}$ films for PV application could make use of this base. There is high scientific and technical merit in conducting work on: 1) innovative ways to control nucleation site density (to control grain size); and, 2) methods to epitaxially grow poly si films on thin poly-Si seedlayers, for example, explosively crystalized thin-films. This epitaxial growth process must occur at a ( 1 ow enough) temperature to be compatible with (low cost) substrate.

\section{Silicon Materials}

Studies are needed on cost effective processes for the removal of impurities and defects from starting materials, since it is more effective to eliminate them from starting material than to remove them later in the process. Defect elimination and control is best performed in collaboration with materials growers and processors. It should be recognized that each growth-fabrication technology has its own unique defect and impurity probiems.

Review Topic 8 - Crystalline Silicon PV Devices and Processing

The industry needs to gain fundamental understanding of effects which influence the manufacturing processes for which they apply. The lack of available knowledge lies in the following areas:

\section{Mechanical Properties of Si Wafers}

Future trends in crystalline $\mathrm{Si}$ manufacturing are towards wafer technologies which are less than 250 micrometers thick. In order to maintain the requisite high manufacturing yields, the mechanisms which control wafer breakage and edge chipping must be understood. Achievement of high mechanical yields is important because it strongly impacts the Si manufacturing costs.

\section{Si Wire-Saw Wafer-sticing Technologies}

Wire saws have substantially improved $\mathrm{Si}$ slicing technology because an entire ingot is sliced simultaneously and the kerf loss is reduced. The present wire saw technology has evolved empiricaliy without sufficient understanding of the mechanisms which control the physics of $\mathrm{Si}$ slicing and the interactions between the wire, the abrasive containing slurry, and the $\mathrm{Si}$ ingot. Process development for recycling the slurry is also essential to reduce costs.

\section{High-Throughput Device Processing Improvements}

The present research efforts need to be accelerated in the areas of rapid thermal processing (RTP) and optical processing. These efforts need to address the basic mechanisms which control these processes and the processing effects on device performance and material properties before they can be transferred to industry.

Integration of High-Efficiency Process and Device Features into Cost-Effective Commercial Technologies

Demonstration of cost-effective $\mathrm{Si}$ device structures and process features remains a high priority of common interest for $\mathrm{Cz}$ and multicrystalline devices. This includes cost-effective implementation of back-surface fields, anti-reflection coatings, surface texture, and advanced fine- 
line screen printing technologies.

\section{Defect Engineering in PV Silicon}

Fundamental research on the interaction between defects and metal impurities including their effect on device performance merits attention. Mechanisms of gettering and passivation of these defects need to be understood. Understanding the role of $H$ passivation during optical/RTA processing is important.

Non-destructive Tools for Si Wafer Characterization in a PV Processing Line

There is an urgent need to develop non-destructive testing tools which can measure important materia7 parameters such as excess carrier lifetime and resistivity as the silicon wafers go through a process line. The tools should be capable of probing the wafers at several points with high testing throughput. This would eliminate adding processing costs to wafers which would not yield devices of acceptable performance.

Feedstock for Crystalline si Photovoltaics

The effects of selected critical impurities on material structure and device performance need to be confirmed in order to develop specifications for solar grade Si feedstock. A thorough assessment of potential existing and promising $\mathrm{Si}$ feedstock production technologies is required.

Review Topic 9 - Amorphous Silicon Material Characterization and Metastability

A number of new directions for amorphous silicon research could be undertaken to address the outstanding needs of amorphous silicon photovoltaic technology. Particular emphasis for new directions should be in the areas of stability, characterization, and deposition. Stability remains the outstanding limitation of amorphous silicon technology despite extensive previous effort. The new directions recommended below could lead to an enhanced understanding and control of metastability. New directions in characterization remain important since much about the structural and electronic properties remain poorly understood. Finally, new directions in deposition are essential since it is clear that the standard glow discharge methods in widespread use do not produce material of sufficient quality at high deposition rates for future needs. Detailed recommendations in these three broad areas are enumerated below.

\section{Stability}

Research activities should be focused on factors affecting metastability. Past research programs have expended significant effort to understand light-induced degradation, primarily studying metastability existing within the standard glow discharge material. Greater emphas is should be placed on research which modifies the metastability of films. Such approaches not only provide improved understanding of its origin but also directly lead to ways to control it.

Specific suggestions for research directions that modify the metastable properties include: 1) changes in the amorphous silicon material, and 2) the study of metastability in related materials. In the first category, the effect of changes in impurity concentrations, alloying, the introduction of new impurities, 
and the alteration of deposition conditions would help to finally resolve outstanding controversies concerning the causes of metastability. In the latter category, the study of metastability in hydrogenated polycrystalline silicon, porous silicon, the crystalline insulator/silicon interface, and microcrystalline silicon could place significant constraints on possible mechanisms for metastability and perhaps lead to a more global perspective on this issue.

\section{Characterization}

In order to clarify the relation between the nanostructure, its dynamics, and the electronic performance and stability, several high resolution studies at the 3$500 \AA$ scale are needed. These should include nuclear magnetic and muon spin resonance which probe local motion and dynamics, atomic force microscopy, sma11-angle neutron and synchrotron $x$-ray scattering, and diffuse $x$-ray scattering, all of which clarify the nanostructural details. These state-of-the-art structural studies should be aimed at both the bulk and interfaces of device structures. Electronic characterization would benefit from a p p o a ches such a s photoconductivity-detected magnetic resonance of devices.

\section{Deposition Issues}

Exhaustive studies of materials and devices fabricated by the standard glow-discharge (GD) method have been performed over many years. Insufficient effort has been expended on novel deposition techniques. The interesting recent results on the hot-wire (at NREL), high-frequency glow-discharge (in Europe), pulsed rf-plasma (in
Japan), and high-temperature, lowpressure GD (in Japan) techniques justify additional investment in these methods and exploratory efforts on totally new approaches. It is important that these new approaches concentrate on producing the films at high deposition rates. Furthermore, they should also focus on reducing the particulate formation during the growth of the film. Replacement of silane by safe sources is desirable.

In parallel with the exploration of new techniques, other device structures such as graded interfaces, gated diodes, and films deposited on textured substrates merit study.

In situ optical characterization to monitor and control the deposition process, which would include issues such as particulates in the plasma and tools such as real-time optical monitoring, should be employed whenever possible.

Review Topic 10 - Amorphous Silicon Fabrication and Characterization

Recent progress in amorphous siliconbased thin-film solar-cell technology has been steady. The research area would benefit from expanded research efforts in materials stability, novel materials approaches, and textured transparent conductors for maximum utilization of sunlight.

\section{Stability}

Light-induced degradation of solar cells remains a key problem. A more intensive effort to understand and characterize device stability and its relationship to materials properties is needed. Diverse high-risk efforts to develop more stable materials are essential since they might lead to new approaches which will enable 
INTERNAL USE ONLY

amorphous thin-film devices to meet the DOE goal of 15 percent commercial modules. Studies such as the existing ECR plasma and hot-wire deposition ones, which shed light on growth processes, may also lead to improved understanding of more conventional materials. To make new materials efforts more effective, a set of standardized opto-electronic characterization techniques should be developed.

\section{Textured Transparent Conductors}

Progress in textured transparent conductors can Tead to significant improvement in short circuit current. Higher carrier mobility leads to better sheet conductance for a given level of free carrier absorption loss. Optimized texturing will lead to improved light trapping. A number of interesting transparent conductors have been introduced recently; they call for more focused work on electronic and structural properties as well as optics and modeling of rough surfaces. High-mobility transparent conductors are now grown at high temperature $\left(>400^{\circ} \mathrm{C}\right)$; therefore, they cannot be deposited on top of amorphous silicon without degradation of the active layers. Focused work on development of commercially viable low-temperature deposition and processing techniques is needed to address this problem.
Review Topic 11 - Technology Development of Polycrystalline ThinFilm PV Devices and Modules

\section{Encapsulation/Packaging for Thin-Film} Solar Cells

Currently, thin-film solar cells employ packaging and encapsulation techniques borrowed from crystalline silicon solar cell technology. However, the materials employed in thin-film modules are very different both physically and chemically from those used in modules incorporating crystalline silicon cells. According7y, the importance of keeping moisture away from the active materials in thin-film modules and the presence or absence of encapsulant degradation products, such as acetic acid from EVA, pose unique problems for thin-film modules. Also, for CdTe modules, glass superstrates are typically empioyed whereas CIS modules are usually formed on glass substrates. With superstrate construction, there is no need for a transparent encapsulant material, opening the range of possibilities for encapsulants. Development of encapsulation/packaging techniques specifically for thin-film solar cells is needed. This program would examine alternative materials as encapsulants and edge seals for use with thin-film modules. Modules would be prepared from thin-film cells from several manufacturers, and life testing carried out under accelerated conditions to define the optimum materials and processes for encapsulation and packaging of these unique materials. 
Accelerated Testing and Degradation Mechanisms

As the thin-film modules enter the commercial market, the need for improved ability to reliably forecast long-term performance becomes critical. The paradigm for this effort was established by the module reliability research conducted by JPL for the silicon module buys of the 1970's. Protocols must be established for real-time exposure testing and fundamental knowledge acquired from which to develop accelerated testing procedures.

The available knowledge has established the CuInSe ${ }_{2}$ junction as inherently stable but, as evidenced by the history of Si modules, this does not ensure stability of an encapsulated 7 arge area module. As field failures inevitably occur, a program of failure anaiysis is needed to accumulate knowledge of the common failure modes. With this information, the necessary basic studies can be initiated to fully characterize the mechanisms of failure and develop remedial structure and process changes. The approach to accelerated testing can be improved as the failure mechanisms are identified.

The situation with CdTe is quite different in that there are numerous reports of instability, generaliy associated with the back ohmic contact to CdTe. For this material system, a fundamental study of the causes of this instability should be initiated immediately. Accelerated testing procedures can then be developed in a manner analogous to the CuInSe ${ }_{2}$ program.
Thin-Film Polycrystalline Silicon on Substrates

Considerable new interest has recently evolved (over 20 presentations at the recent Hawaii conference) in the area of thin $(<50 \mathrm{c})$ micro- and poly-crystalline silicon materials deposited onto low cost substrates. A lack of U.S. research in this area would be an oversight.

In particular, lower temperature processes are worthy of investigation because substrate requirements are considerably relaxed. Low temperature processes allow the incorporation of device designs that include light trapping and insulating substrates (for monolithic module fabrication) which are essential to meeting $D O E^{\prime} s$ long-term efficiency goats.

\section{Additional Materials R\&D}

The current projects' relative emphasis on absorber layer development and limited or nonexistent attention to substrate, contact, interface, and transparent conductor development begs for balance. As industry drives to scale-up specific thin-film technologies, issues affecting production yields will emerge. In response to these challenges, a number of alternative materials and processes may be needed to support the fundamental absorber layer production technology. Few choices exist at present because little effort has been applied to expand the options.

Substrate Options: Soda-7ime glass is the standard starting material for both $\mathrm{CuInSe}_{2}$ and CdTe. There are at least two immediate areas meriting investigation. These concern 
composition and mechanical properties. With regard to composition, controlling the sodium content of the active absorber film may offer potential enhancements for CuInSe ${ }_{2}$ performance optimization. In addition, higher temperature compatibility for low cost glass could widen the process window for formation of alloys of CuInSe $\mathrm{C}_{2}$, especialiy for sulfur addition. With respect to physical properties, options for enhancing glass strength could permit NREL's evaporation process for high efficiency $\mathrm{CuInSe}_{2}$ to be rapidly scaled to commercially significant levels.

Contact 0ptions: A range of possible back contacts for CdTe is still lacking after many years. In addition, no alternatives to molybdenum for the $\mathrm{CuInSe}_{2}$ back contact have received significant attention. An expansion of contact choices would be beneficial to both technologies. Additional materials would create broadened manufacturing processing tolerance.

Transparent Conductor Options: The glass industry has assumed the major responsibility for tin oxide development not only for CdTe but al so amorphous silicon. At present, options for device enhancement are constrained by the supplier's willingness to participate in a collaborative effort. This limitation would be reduced with a tin oxide research effort.

Zinc oxide's wide-band transmission and relatively good conductivity have not been integrated with fullyoptimized deposition equipment. Some additional effort here could define the potential for both new material and device configurations, perhaps leading to wider production choices.
Explanation of Properties as Related to Performance for Polycrystalline Solar Cells

Obtaining fundamental data on typically rough polycrystalline films of CIS is difficult and expensive. Having a smooth, specular CIS surface would allow easier and more accurate measurement of the interaction between various window/junction characteristics.

Thin-film, Targe grain CIS layers can be grown on single crystal, polished GaAs wafers in an evaporator. This technique for making samples would be much simpler and quicker than trying to grow various single crystal ingots and make slices. This smooth CIS absorber layer may then be used to explore the effect of various CdS and/or other high bandgap window layers that might be of interest.

Comparable approaches could probably be used for CdTe junction and contact studies.

\section{Review Topic 12 - Device Fabrication and Analysis of CIS-Based Materials}

\section{Junctions}

After more than a decade in developing CIS-based solar cells, basic knowledge concerning the junction of the device is still lacking. A clear determination of what the device junction is, a true heterojunction (CdS/CIS) or a buried heterojunction (buffer/VOC/CIS), is needed. If the buried heterojunction assumption is true, what is the role of the buffer layer in making a good cell and how does one determine the physical requirements (e.g., bandgap and electron affinity) of the buffer layer? If the device is a true heterojunction, why is CdS the best partner? From the results of this 
sort of study, a better physical model to describe the device can be developed and a better choice of a buffer (or n-type partner) layer other than CdS can be made.

CdS is known to be an important compound for thin-film solar cells because CdS layers improve overall cell efficiency. Specifically, and most visibly, use of CdS improves the curve shape best recognized in an improved fill factor. This could be interpreted to be the result of the well-known ability of CdS to limit the field in junctions to below $50 \mathrm{kV} / \mathrm{cm}$, i.e., below tunneling fields by "field quenching" (emission of holes from Coulomb-attractive hole traps). Such a field limitation is essential in junctions within materials with defect densities above $10^{16} \mathrm{~cm}^{-3}$.

It is essential to: 1) validate this hypothesis and to propose an explanation for reducing junction leakage via tunneling; and 2) to find other materials which have similar properties in order to provide alternative materials that may make even better partners for thin-film solar cells (less lattice mismatch, less toxicity, etc.).

\section{Manufacturability}

The manufacturability of CIS PV technology is a key determinant in the overall success of the DOE's thin-film PV Program. Issues of manufacturability that require additional emphasis in future research priorities include: 1) interactions of the substrate with film formation and junction performance; 2) formation of stable, low-loss monolithic electrical interconnects; 3) manufacturing issues of CIGS formation process such as materials-use efficiency, spatial scalability, deposition rate, process temporal stability, degree of process control, and device reproducibility; and 4) opportunities for simplified and/or cheaper manufacturing such as superstrate structures and simple CIGS formation processes. - Prioritization of manufacturability issues should emphasize near-term requirements over long-term opportunities. Activities in manufacturability should focus on the pragmatic implementation of laboratory advances with the goal of accelerating thin-film CIS pilot production.

\section{Photoelectronic Properties of CIS}

The fundamental photoelectronic properties of CIS that determine the overa11 PV device characteristics are those related to free carrier photogeneration, transport, and recombination. Generally speaking, these include: 1) drift mobilities, 2) photogeneration efficiencies, 3) recombination rates, and 4) diffusion length. Techniques for measuring the parameters are we11 known. These parameters can then be related to the chemical pathway used in the materials preparation (e.g., temperatures and components). By appropriate models, the PV device characteristics can be related to the same parameters and thus to stages in the chemical pathway used for sample preparation. These techniques offer a means by which: 1) limitations to future improvements in device performance can be identified, and 2) quality control of the manufacturing process can be established on a quantitative basis.

\section{Back Electrode}

Poly-crystaliine molybdenum is known as an acceptable back electrode for CIS solar cells. However, it has a number of disadvantages, including 
its crystallinity. It is an insufficient diffusion barrier to alkali-diffusion from the underlying soda-lime glass, and it has Timited wetability. Amorphous substrates have advantages for desirable CIS growth.

Research for an alternate back electrode is suggested. This could be molybdenum-related but with changing of its surface topography, e.g., by low temperature deposition (quasi-amorphous structure) or by sufficient surface roughening via gas discharge or other particle bombardment. Other changes could involve thin "amorphous" interlayers that also act as diffusion barriers and have appropriate work functions to yield a desirable ohmic contact to CIS.

The goal is an electrode that does not interfere with appropriate CIS crystal growth and avoids peeling, pinhole formation, electrical degradation, and other effects that are detrimental to cell manufacturability, cell life, and cell performance.

Two kinds of models for these electrodes are important: 1) growth process models, and 2) transport property models on recombination. For the growth processes, modeling of the diffusion processes for different elements in CIS are crucial in understanding state-ofthe-art growth techniques. For high efficiency PV devices using CIGS, the roles played by traps and graded-conduction-band edge on electron-hole recombination are essential in understanding the recombination rates and in the future development of more efficient devices. This kind of modeling involves the development of physicaliy plausible models with realistic interacting strengths.

\section{Diode Models}

Consumer-friendly software is needed for basic physics photo-diode modeling, involving realistic transport-, continuity-, and Poissonequations for two-carrier models. It could be used at the bench to replace potentially misleading network models involving resistances and diodes.

\section{Tandem Cel1s}

None of the projects evaluated investigated the possibility of tandem (cascade) solar arrays based on CIS low-bandgap bottom cells. Such an approach has the potential for producing considerably higher efficiency at the array level, which could lead to considerable advances toward the DOE goal of cost-effective electricity generation. Since CIS is currently a substrate technology, and CdTe is currently a superstrate technology, one obvious choice is to produce modules by adhering a CdTe superstrate to a CIS substrate in a 4-terminal configuration. An alternative, technologically riskier approach would be to produce monolithic tandem cells by depositing the wide-bandgap cell directly onto the low-bandgap CIS cell, or vice versa. This approach requires that both high- and low- bandgap cells be produced by either the superstrate or substrate technology and to solve issues related to interdiffusion and reaction of materials at the high temperatures required for deposition.

An alternative approach to thin-film tandems is to remain within the I-II$\mathrm{VII}_{2}$ semiconductor system. This would require development of widebandgap semiconductors within the chalcopyrite compounds, such as $\mathrm{CuGaSe}_{2}$ or $\mathrm{CuInS}_{2}$, and the concurrent development of high transparency heterojunction window materials. 


\section{Superstrate Cells}

Development of CIS-based cells in a superstrate configuration, as opposed to the current substrate configurations, could have some benefits to commercial array manufacture. A superstrate module would use the same glass sheet for encapsulation as for growth, potentially lowering cost. It would transfer the problems of adhesion from the glass/Mo/CIS interface to a glass/transparent conductor/CIS interface, which could be either advantageous or detrimental. It would also allow some additional freedom in choice of back contact material. A superstrate technology would also be a simpler configuration on which to produce monolithic interconnections and would leverage interconnect technology already developed for amorphous silicon. Finally, a superstrate CIS technology leads to the possibility of integration with CdTe technology to produce monolithic tandem cells.

Review Topic 13 - Analysis, Characterization, and Hodeling of Thin-Film Materials and Devices

The future research opportunities that exist in the area of analysis, characterization, and modeling of thin-film materials and devices can be categorized into two broad categories: structural and optoelectronic.

In the structural area there is a need to investigate: a) the grain structure of the material; b) kinetic processes such as diffusional instabilities that may be present in the completed devices, formation of metastable phases and stability of the back and front contacts; and c) junction physics and chemistry.

In the opto-electronic area, doping distribution in 3-D, diffusion length distribution in 2-D, differentiation of electronic and lattice relaxation, and the potential of band gap engineering should be investigated.

Research in the above areas could significantly contribute to achieving the stable cell efficiency goals of the Photovoltaics Program.

Review Topic 15 - Exploratory, Advanced, and High-Efficiency Materials

\section{Dye-Sensitized Nanostructured Materials for PV Applications}

A photovoltaic device based on dyesensitized nanocrystalline semiconductor particles promises to meet the goal of efficient, low-cost solar energy conversion. To develop this concept further, research efforts are needed in the areas of rational design of dye molecules capable of fast charge injection into $\mathrm{TiO}_{2}$, alternative material candidates to $\mathrm{TiO}_{2}$, redox electrolytes with fast mass and charge transport properties, and a good understanding of the electrode structure and materials properties.

Multi-component nanostructured materials offer another avenue of opportunity in this area. Candidate structures are $\mathrm{TiO}_{2} / \mathrm{CdSe}$, $\mathrm{SnO}_{2} / \mathrm{TiO}_{2} / \mathrm{CdSe}$, etc. The issues of efficient change separation and recertification of back-electron transfer can be effectively addressed with these structures. In this regard, these nanostructured systems provide good models for artificial photosynthetic analogs. 
Exploration of Organic Materials for PV Applications by UltrastructureControl Synthes is Methods

A priority activity is the exploration of organic materials for the fabrication of PV cells. Motivation is provided by the we11known photoconversion efficiencies of biological organic materials and the large oscillator strengths of organics. Deficiencies include problems with exciton diffusion, charge carrier generation, and carrier transport. While organic polymers and organics (and organometallic) multiple quantum well structures hold some promise for $1 \mathrm{ight}$-emitting diode applications, the requirements for PV cell applications are more stringent than those for LED applications.

To gain a realistic assessment of the potential of organics for PV applications, studies of organic materials prepared by synthetic methods which lead to structurally well-defined materials are recommended. In this regard, organic molecular beam deposition fabrication of well-defined, low defect structures is endorsed. Thin film fabrication by organic sequential synthesis methods, including methods involving electroactive polymers, is also of interest.

In summary, planar organic and organometallic materials possess diffuse pi orbitals and can be organized into structures which lead to quantum confinement. Providing that precise control of molecular ultrastructure engineering is maintained, spectroscopic and electrical transport measurements should permit determination as to whether or not meaningfuT PV efficiencies can be realized with organic and organometallic materials.

\section{Wider Range of Photovoltaic Semiconductors, Including Wide- bandgap Semiconductors}

Advances in growth and processing techniques are extending the range of semiconductor materials that may have application in many different arenas. For example, wide-bandgap semiconductors like GaN, SiC, and diamond are currently offering exciting prospects for blueultraviolet lasers, high temperature/high power electronics, and blue-ultraviolet detectors. If growth and fabrication problems can be overcome, it is conceivable that tandem cells involving wide-bandgap materials may lead to enhanced efficiencies. In any event, these robust materials could provide options for either large-scale or niche applications. There are literally hundreds of other semiconducting materials that may be worth studying in the exploratory spirit of this program. 
APPENDIX A

PROJECT TITLES

1. "Silicon-Film Photovoltaic Manufacturing Technology"

2. "Continuous Roll-to-Roll Amorphous Silicon (a-Si) Photovoltaic Manufacturing Technology"

3. "Photovoltaic Manufacturing Technology (PVMaT) Improvements for ENTECH'S Concentrator Module"

4. "Photovoltaic CZ Silicon Manufacturing Technology Improvements"

5. "Large Area Multijunction Amorphous Silicon Alloy Production"

6. "Amorphous Silicon PVMaT - Phase 2A"

7. "Amorphous Silicon Manufacturing Technology - Phase IIa"

8. "Automated Solar Cell AssembTy Teamed Process Research"

9. "Photovoltaic Manufacturing Technology (PVMaT): Advanced Development of PV Encapsulants"

10. "Photovoltaic Cel1 and Module Efficiency Measurements"

11. "Photovoltaic Solar Radiometric Measurements and Evaluation"

12. "PV Module Qualification and Exploratory Testing, Test Method Development and Failure Analysis"

13. "PV Module Materials ard Component Reliability"

14. "Photovoltaic Cell and Module Evaluation"

15. "Photovoltaic Module Development"

16. "Commercialization of the SEA Powergrid"

17. "Research on Stable, High-Efficiency Amorphous Silicon Multijunction Modules"

18. "Research on Amorphous Silicon-Based Thin-Film Photovoltaic Devices"

19. "Processing and Modeling Issues for Thin-Film Solar Cell Devices"

20. "Comprehensive Research on Stability and Performance of a-Si:H and Alloys"

21. "Amorphous Silicon Devices and Device Characterization" 
22. "Optimization of Transparent and Reflecting Electrodes for Amorphous Silicon Solar Cells"

23. "Photovoltaics for Utility Scale Application (PVUSA)"

24. "Modu? and System Performance"

25. "Amorphous Silicon Utility/Industry PV Power Project"

26. "PV Standards and Codes"

27. "Photovoltaic (PV) Systems Engineering"

28. "Tests, Evaluation and Application of Photovoltaics Technology and Charge Controller Evaluation"

29. "PV Systems Field Evaluation"

30. "Photovoltaic Balance of Systems"

31. "Development of the RFPCD Technique for the Measurement of MinorityCarrier Lifetime in Semiconductors"

32. "Thin-Film Materials Development Using Time-Resolved Photoluminescence as a Diagnostic Tool"

33. "Applications of the Scanning Defect Mapping System for Characterizing Solar Cell Materials and Devices"

34. "Scanning Electron and Optical Beam Characterization of PV Devices"

35. "Atomic and Nanoscale Investigations and Development of Photovoltaic Semiconductors Using Scanning Probe Microscopies"

36. "Room-Temperature FT-Luminescence Analysis of $\mathrm{Cu}(\mathrm{In}, \mathrm{Ga}) \mathrm{Se}_{2}$ Films and Devices"

37. "Quantification of Transmission Electron Diffraction (TED) Measurements in the TEM"

38. "Crystalline-Silicon Cell Development"

39. "Crystalline-Silicon Process Development"

40. "Advanced Processing of Solar Grade Crystalline Silicon"

41. "University Center of Excellence in Photovoltaic Research and Education: Crystalline Silicon Photovoltaic Technology"

42. "Effects of Aluminum Gettering and Forming Gas Anneal on Multicrystalline Silicon Solar Cells" 
43. "Process Optimization of Silicon Spherals Using RFPCD as an On-Line Diagnostic Tool"

44. "Defect/Impurity Effects on Minority Carrier Lifetime and Diagnostic PV Cel1 Performance in Silicon"

45. "Development of Optical Processing and Hydrogenation for Fabrication of Low-Cost, High Efficiency Silicon Solar Ce11s"

46. "Optimization of Gettering Processes for Photovoltaic Silicon"

47. "Theory of Electronic Structure of CuInSe $e_{2}$ and Related Compounds"

48. "Fundamental Studies of the Effect of Crystal Defects on CuInSe $2 / \mathrm{CdS}$ Heterojunction Behavior"

49. "Surface Analytical Characterization of the Chemistry and Electronic Structure of Cu(In,Ga)Se $e_{2}$ Thin-Film Homojunction Devices"

50. "Qualitative and Quantitative Analys is of Multilayer Polycrystalline ThinFilm Materials"

51. "Optical Characterization of Defect States in CIS and CIGS Semiconductors"

52. "Device Physics of Thin-Film Polycrystalline Cells and Modules"

53. "Processing and Modeling Issues for Thin-Film Solar Cell Devices"

54. "Development and Application of a Computer Model for CdTe and CIS Based Solar Cells"

55. Photovoltaic Design Assistance Center - "Government Applications"

56. Photovoltaic Design Assistance Center - "Utility Applications"

57. Photovoltaic Design Assistance Center - "International Applications"

58. "International Rural Electrification: Joint U. S./Brazil Project"

59. "Cost Effective PV for Rural and Municipal Applications"

60. "Utility Photovoltaic Group"

61. "Investigation of Polycrystalline Thin-Film CIS Solar Ce1ls Based on ZnSe Windows"

62. "Process and Modeling Issues for Thin-Film Solar Cell Devices (CuInSe $e_{2}$ Based Thin-Film Solar Cells)"

63. "Heterojunction Development and Optimization in Thin-Film Compound Semiconductor Solar Cells" 
64. "Polycrystalline CuInSe ${ }_{2}$ (CIS) and CdTe Photovoltaic Solar Cells"

65. "High-Efficiency Thin-Film CIGS PV Devices: Progress Towards a Universal Approach to Absorber Fabrication"

66. "World Record High-Efficiency CIGS Solar Cells: Fabrication Process and Projection on Manufacturability"

67. "Growth Mechanisms of the CIGS Absorber Responsible for the High Efficiency"

68. "CdTe Device Development and Materials Optimization: An Investigation of the CdTe/CdS Interface"

69. "PoTycrystalline p-CdTe Contact Development"

70. "CdS/CdTe Heterojunction Solar Ce11s: Their Composition, Chemistry, and Electronic Structure Studied with Electron Spectroscopy"

71. "Structural and Luminescence Characterization of CdTe Thin Films"

72. "Processing and Modeling Issues for Thin-Film Solar Cell Devices (CdTeBased Thin-Film Solar Cells)"

73. "Polycrystalline Thin-Film Cadmium Telluride Solar Cells Fabricated by Electrodeposition"

74. "High-Efficiency, Thin-Film Cadmium Telluride-Based Solar Cells; Thin-Film CdTe PV Cel1s"

75. "Advanced Processing Technology for High-Efficiency Thin-Film CdTe Solar Ce11s"

76. "Advanced Processing Technology for High Efficiency. Thin-FiTm CuInSe $e_{2}$ and CdTe Solar Cells"

77. "High-Efficiency III-IV Solar Cells"

78. "Ordering in III-V Alloys"

79. "Optical Spectroscopic Investigations of Spontaneous Ordering in GaInP"

80. "Solid-State Theory Studies of PV Semiconductors"

81. "Solar Energy Conversion at Dye Sensitized Nanostructured Electrodes Fabricated by Sol-Gel Processing"

82. "Very High Efficiency PV Celis Based on Fully Organic Multiple Quantum Wel1s"

83. "High Efficiency III-IV Materials Development Using Time-Resolved 
Photoluminescence as a Diagnostic Tool"

84. "Rooftop PV System"

85. "Development of the AC Photovoltaic Module (Phase 1 of Building Opportunities in the U.S. for Photovoltaics)"

86. "Development of a Dispatchable PV Peak Shaving System (PV: BONUS, Phase I)"

87. "Evaluation of DSM Incentive Opportunities for Photovoltaics"

88. "Solar Resource Utility Load Matching Assessment"

89. "Design for Photovoltaics: Curriculum Development for North American Architecture School Faculty"

90. "PV-Integrated Modular Homes"

91. "Detailed Non-contact Electrical and Structural Characterization of Photovoltaic Silicon Substrates"

92. "Trace Element Analysis by SIMS in Poly- and Single-Crystalline Silicon Materials"

93. "Identification and Control of Lifetime-Reducing Defects in Polycrystalline Silicon Photovoltaic Materials"

94. "Diffusion-Segregation Equation for Silicon Process Modeling"

95. "The Role of Point Defects and Impurities in the Processing Performance of Crystalline Silicon Solar Cells"

96. "Thin-Layer Silicon Growth from Metal Solutions for Low-Cost PV Device Structures"

97. "Thin-Film Silicon by Constitutional Supercooling"

98. "High Performance Porous Silicon Solar Cel] Development"

99. "Electronic Processes in Thin-Film PV Materials"

100. "Smal1-Angle and X-ray Scattering Studies of Microvoids in Amorphous Silicon-Based Semiconductors"

101. "Experimental Study of Photodegradation in a-Si:H Solar Cells"

102. "Microscopic Origins of Metastable Effects in a-Si:H and Deep Defect Characterization in the a-Si,Ge:H Alloys"

103. "Defects and Transport in Amorphous Silicon Based Semiconductors" 
104. "Growth Mechanisms and Characterization of Hydrogenated Amorphous Silicon and Germanium Films"

105. "In-Situ Characterization of Growth and Interfaces in a-Si:H Devices"

106. "Hydrogen, Metastability, and Electronic Defects in Amorphous Silicon"

107. "New Deposition Methods for Amorphous Silịcon"

108. "Research on Polycrystalline Thin-Film Submodule Based on CuInSe Materials"

109. "Non- $\mathrm{H}_{2} \mathrm{Se}$, U7tra-Thin CIS Devices"

110. "Nove? Two-Stage Selenization Methods for Fabrication of Thin-Film CIS Cells and Submodules"

111. "Research on High-Efficiency, Large-Area CuInSe (CIS)-Based Thin-Film Modules"

112. "Fabrication of Stable, Large-Area, Thin-Film Cadmium Telluride Photovoltaic Modules"

113. "Development of Large-Area Monolithically Integrated Silicon-Film Photovoltaic Modules"

114. "Module Process Optimization and Device Efficiency Improvement for Stable, Low-Cost, Large-Area, Cadmium Telluride-Based, Photovoltaic Module Production"

115. "Health and Environmental Effects of Photovoltaic Energy and Safety Assistance Center" 
Abetes, Dr. Benjamin

Exxon Research and

Engineering Company

Allen, Professor Roland E.

Texas A\&M University

Amick, Dr. James A.

Consultant

Ast, Professor Dieter G.

Corne11 University

Bailey, Dr. Sheila G.

NASA Lewis Research Center

Ba11, Dr. Timothy J.

Applied Power Corporation

Bel1, Dr. Richard 0.

Consultant

Benatar, Dr. Lisa E.

Park Scientific Instruments

Benson, Dr. Kenneth

Consultant

Böer, Professor Karl W.

University of Delaware

Borrego, Professor José M.

Rensselaer Polytechnic Institute

Borsenberger, Dr. Paul M.

Eastman Kodak Company

Brenneman, Dr. Robert K.

Elkem Metals Company

Chadi, Dr. James D.

NEC Research Institute, Inc.

Chalmers, Dr. Steve

Consultant
Cheek, Dr. Gary

Analog Devices

Che]ikowsky, Professor James R.

University of Minnesota

Chen, Dr. W. S.

Consultant

Cheung, Dr. Jeffrey

Rockwe11 International

Chu, Dr. Shirley S.

Consultant

Chu, Professor Ting L.

Consultant

Cody, Dr. George D.

Exxon Research \& Engineering Co.

Combs, Dr. Chuck

NavaT Air Weapons Station

D'Aiello, Dr. Robert V.

Consultant

Dalton, Professor Larry

University of Southern California

Dalton, Mr. John

Consultant

de Neufville, Dr. John P.

Voltaix, Inc.

DeMeo, Dr. Edgar A.

Electric Power Research Institute

Dillington, Dr. David L

Spectrolab, Inc.

DiNardo, Professor N. John

Drexe1 University

Ducey, Dr. Roch A.

US Army Corps of Engineers 
Eberspacher, Dr. Chris

Unisun

Eser, Dr. Erten

Energy \& Environmental Systems, Inc.

Fahrenbruch, Professor A1 an L.

Stanford University

Flood, Dr. Dennis

NASA Lewis Research Cente

Fong, Dr. Ching-Yao

University of California, Davis

Fortmann, Professor Charles M.

Pennsylvania State University

Fritzsche, Professor Hellmut

The University of Chicago

Gajdardziska-Josifovska, Prof.

Marija

University of Wisconsin

Garcia III, Dr. Alexander,

The Aerospace Corporation

Gay, Dr. Charles F.

Unisun

Goodman, Jr., Dr. Frank R.

Electric Power Research Institute

Griffith, Mr. Frank W.

Consultant

Hovel, Dr. Harold J.

IBM Corporation

Hurd, Mr. Jeffery L.

IBM Corporation

Jackson, Dr. Warren B.

Xerox Palo Alto Research Center

Jackson, Professor Kenneth A.

University of Arizona

Jennings, Ms. Christina

Pacific Gas \& Electric
Kakalios, Professor James

University of Minnesota

Kamat, Professor Prashant V.

University of Notre Dame

Kanicki, Dr. Jerzy

University of Michigan

Kardauskas, Dr. Michae 1

ASE Americans, Inc.

Kaszeta, Dr. William J.

Photovoltaic Resources International

King, Mr. J. Willard

JWK Associates, Inc.

KTeinman, Professor Leonard

The University of Texas at Austin

KTemchuk, Dr. Peter

Consultant

Landis, Dr. Geoffrey A.

NASA Lewis Research Center

Mahajan, Professor S.

Carnegie Mellon University

Marvin, Dr. Dean C.

The Aerospace Corporation

Meakin, Professor John D.

University of Delaware

Menendez, Professor Jose

Arizona State University

Miller, Dr. Jeffrey N.

Hewlett-Packard Labs

Mishra, Dr. Kamal

MEMC Electronic Materials, Inc.

Mitche11, Dr. Kim

Siemens Solar Industries

Neugrosche1, Professor Arnost

University of Florida 
Noe1, Professor Gerald T.

Central State Unversity

Persans, Dr. Peter D.

Rensselaer Polytechnic Institute

Peterson, Dr. Terry

Electric Power Research Institute

Rahman, Professor Saifur

Virginia Polytechnic Institute

Ranjan Das, Dr. Suhit

Institute for Microstructural

Sciences

Redfield, Professor David

Stanford University

Risser, Dr. Vern

Daystar Incorporated

Roberts, Dr. Donald R.

FuelCell Corporation of America

Rose, Mr. Charles M.

Fue 1 Cell Corporation

of America

Rosey, Mr. Richard

EBARA Solar, Inc.

Ruda, Professor Harry E.

University of Toronto

Russe11, Dr. Paul E.

Power Quality Engineering, Inc.

Sandana, Dr. Devendra K.

IBM Research Division

Thomas J. Watson Research Center

Schumacher, Dr. John

La Costa Materials Technology

Shibib, Dr. M. Ayman

AT\&T-ME

Shiner, Professor Joe

Iowa State Univeersity
Singh, Dr. Pritpal

Villanova University

Sinton, Dr. Ronald A.

Sinton Consulting

Sivananthan, Dr. Sivalingam

University of Illinois

Smith, Dr. Darryl L.

Los Alamos National Laboratory

Soinski, Dr. Arthur J.

Consultant

Stavola, Professor Michael J.

Lehigh University

Stefanakos, Professor Elias K.

The University of South Florida

Tiedje, Professor Tom

University of British Columbia

Tsai, Dr. Chuang Chuang

Xerox Palo Alto Research Center

Wagner, Dr. Richard

Consultant

Wald, Mr. Fritz V.

ASE Americas, Inc.

Weakliem, Professor Herbert A.

Princeton University

Welch, Dr. James $H$.

Consultant

Wernick, Dr. Jack H.

Consultant

Wieder, Professor Harry $H$.

University of California at San Diego

Holf, Professor Martin

Consultant

Wolford, Professor Don

Iowa State University 
Yacobi, Dr. Ben G.

Ashurst Technology Centre

Yang, Dr. Lin $\mathrm{H}$.

Lawrence Livermore Nationa]

Laboratory 

United States

Department of Energy

Washington, DC 20874-1290

Official Business

\section{ER-5}

